peuvent être transférées temporairement aux liquides et que nous n'avons pu jusqu'alors ni expliquer ni comprendre. La coagulation du lait par la présure est un phénomène biologique, nous dirons même un phénomène vital, que nous produisons in vitro.

\title{
BIBLIOGRAPHIE.
}

[1] O. Hammarsten:

a) Om mjölkystningen och de dervid verksamma fermenterna i magslemhinnan. Upsala läkareföreningens förhandlingar, 1872-73.

b) Om det kemiska forlöppet vid caseinets coagulation med löpe. Upsala läkareförenings förhandlingar, 1873-74.

c) Om lösligt och olösligt casein i mjölken. Upsala läkareförenings förhandlingar, 1875-76.

d) Zur Kenntniss des Kaseins und der Wirkung des Labfermentes. Upsala, 1877.

[2] Ch. Porcher. Le lait au point de vue colloïdal. Lyon, 1929.

[3] E. Duclaux. Le lait. Etudes chimiques et microbiologiques. Paris, 1889.

[4] W. vAN DAM. Sur les enzymes de la présure, et Sur la coagulation du lait par la présure. Revue générale du lait, 1910.

[5] L. L. vaN SLYke. Composition and properties of same Casein and Paracasein compounds. New York State Agricultural Experiment Station Technical Bulletin, $\mathrm{n}^{\circ} 26,1912$.

[6] O. Hammarsten. Lehrbuch der physiologischen Chemie. Wiesbaden : $5^{\mathrm{e}}$ édition, $1904 ; 11^{\mathrm{e}}$ édition, 1926.

[7] Vandelivelde. Sur la classification des protéines du lait. Revue générale du lait, 1910.

[8] L. Lindet et P. Ammann. Divers travaux sur la caséine. Comptes rendus de l'Académie des Sciences : 1906, 1912, 1913.

[9] Chr. Barthel, E. SANdBerg et E. Haglund. Recherches de la présure dans les fromages. Le Lait, 1928.

[10] P. Bary. Où en est lá chimie colloïdale ? Paris, 1927.

\section{LE DOSAGE DE LA MATIÈRE GRASSE DANS LES CASÉINES INDUSTRIELLES}

\section{par}

JEAN PIEN

Ingénieur chimiste (I. C. R.),

Docteur ès sciences,

Directeur des Laboratoires des

"Fermiers Réunis ". et

\section{MARC BERGIER}

Ingénieur agricole (I. A. N.), du Laboratoire des caséines des "Fermiers Réunis"

\section{er ARTICLE}

La teneur en matière grasse des caséines est l'un des facteurs les plus importants de l'appréciation de la qualité de ces substances.

Dans les applications industrielles on relève, au moins, deux 
catégories principales d'inconvénients ou d'accidents dus à des teneurs trop élevées en matière grasse :

10 Diminution du pouvoir adhésif des colles préparées avec la caséine. (En menuiserie, ébénisterie, earrosserie, industrie du contreplaqué, cette circonstance peut devenir désastreuse.)

$2^{\circ}$ Diminution du rendement des colles et enduits. La matière grasse atténue fortement la viscosité des solutions colloïdales de caséine. Pour retrouver une certaine viscosité avec un produit gras on est obligé d'utiliser davantage de colle ou de caséine. L'industriel dit que son " rendement » diminue.

Cet inconvénient d'ordre économique, joint à la difficulté technique précédente, déprécie les caséines contenant une proportion trop élevée de matière grasse.

Il est donc du plus haut intérêt de pouvoir doser cette substance avec exactitude.

Ce qui frappe le plus quand on fait la bibliographie de la question du dosage de la matière grasse dans les caséines, e'est le grand nombre de méthodes ou de variantes d'un même principe, qui ont été proposées par divers auteurs (SHAw, WERNER-ScHMIDT, SçHMIDTBondzynski, Höpener, Tapernoux, Chollet, Fouassier, TeiChert, Schoonjans, Kruger, etc.). En fait, toutes ces méthodes peuvent aisément se grouper en catégories peu nombreuses et, à notre avis, le classement qu'on doit en faire pourrait être le suivant :

\section{PREMIER GROUPE:}

\section{Méthodes où la matière grasse est extraite par l'éther.}

$1^{0}$ L'extraction se fait sur la caséine solide : méthode Soxhlet.

$2^{\circ}$ La caséine est préalablement dissoute :

a) En milieu alcalin :

๔) Apparentée à la méthode Röse-Gottlieb : méthode Shaw [1].

ß) Apparentée à la méthode Meillère : méthode Tapernoux [2].

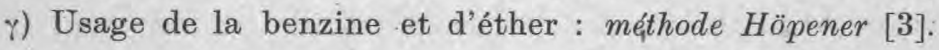

b) En milieu acide : (toutes apparentées à la méthode Gottlieb) :

a) Méthode Schmidt-Bondzynski (sans addition d'alcool) : Modes opératoires Dornic et Daire [4] et Chollet [5].

ß) Méthode Gottlieb-Ratzlaff :

Mode opératoire modifié par FouAssien [6] (avec addition d'alcool). 


\section{DEUXIEME GROUPE:}

\section{Méthodes oủ la matière grasse est séparée à chaud de la solution sulfurique de caséine.}

Ces méthodes sont issues du procédé Gerber.

Citons principalement :

a) Méthode Kruger [7] (dissolution à $70^{\circ}$ ).

b) Méthode Tapernoux [8] (application de la méthode Teichert aux easéines) (dissolution à $85-90^{\circ}$ ) ; déjà préconisée par Schoonjans [9].

Nous n'avons rappelé dans cet essai de classement que les principales méthodes. Cette abondance de travaux et de recherches nous montre bien, déjà, combien la question est délicate et difficile à résoudre. Ainsi que nous le montrerons, plusieurs de ces méthodes donnent des résultats divergents. Il est donc certain que quelquesunes d'entre elles sont inexactes.

Notre but, dans ce travail, est d'essayer de découvrir la ou les méthodes susceptibles d'être retenues, en apportant, si possible, l'explication de l'erreur commise avec celles qui nous paraissent inexactes.

$$
*^{*} *
$$

Après avoir défini le but de notre étude, exposons-en le principe.

Il y avait deux façons d'envisager le problème :

$1^{0} \mathrm{Ou}$ bien appliquer les méthodes précédentes en analysant les extraits obtenus comme nous l'avons fait pour l'étude de l'analyse des laits écrémés [10]; on eût apporté ainsi une preuve des surcharges trouvées dans certaines méthodes, mais sans découvrir aisément l'erreur par défaut commise dans d'autres par insuffisance d'extraction.

$2^{\circ} \mathrm{Ou}$ bien tâcher de fabriquer une caséine à teneur connue en matière grasse, s'arrêter à une méthode donnant sur cette easéine le chiffre théorique prévu (en démontrant d'ailleurs que cette exactitude n'est pas le fruit d'une compensation d'erreurs) et, finalement, comparer les autres méthodes à celle qui aura été ainsi reconnue exacte.

Nous avons choisi ce deuxième principe. Il offre une garantie sérieuse, puisque la découverte de la méthode exacte, de base, est fondée à la fois sur la connaissance a priori de la teneur en matière grasse de la caséine et sur la démonstration directe (par d'autres moyens) de l'exactitude du chiffre trouvé.

L'analyse des extraits éthérés, au lieu de servir de point de départ (comme dans le premier principe utilisé dans notre étude 
sur la matière grasse des laits écrémés), viendra, en fin d'étude, servir d'appoint et de complément de démonstration.

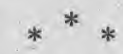

Ainsi, le plan de notre travail se dégage de lui-même.

Nous étudierons successivement : grasse.

$1^{\circ}$ La préparation de caséines à teneur connue en matière

$2^{\circ}$ Le choix de la méthode donnant un chiffre exact, l'exactitude de ce chiffre devant être fondée :

a) sur la similitude avec les chiffres théoriques des caséines mises en cuvre ;

b) sur une démonstration directe.

$3^{\circ}$ L'examen critique des diverses méthodes proposées pour le dosage de la matière grasse des caséines.

Le présent article est l'exposé des deux premières parties du plan. La dernière paraîtra dans un prochain article.

\section{* * * \\ PREMIERE PARTIE. \\ PRÉPARATION DE CASÉINES A TENEUR CONNUE EN MATIËRE GRASSE.}

La pratique industrielle nous a familiarisé avec la très féconde méthode des bilans. Elle nous a donné de si heureux résultats en maintes circonstances que nous n'avons pas hésité à l'appliquer ici.

Le mécanisme, dans le cas particulier qui nous occupe, en est le suivant :

Soit un volume connu de lait écrémé dont on veut faire de la caséine. Toute la matière grasse de ce lait va se répartir en un certain nombre de portions : caillebotte, sérum, parois des récipients... $\mathrm{Si}$ on peut déterminer avec exactitude la matière grasse totale mise en jeu et la matière grasse perdue dans les sérums, eaux de lavage, jus de pressage, récipients, etc., par différence on aura la quantité de matière grasse passée dans la eaillebotte. C'est ainsi que nous avons procédé.

Cette méthode mérite le reproche de donner un chiffre par différence. Il est cependant facile de se rendre compte par le calcul que les causes d'erreur sont faibles (à condition que les dosages soient exacts). Elle permet en tout cas, sur des bilans faits en double, d'arriver à des résultats très voisins; la dispersion des chiffres n'excède guère celle qu'on peut exiger d'une bonne méthode d'analyse directe. Il semble donc, en opérant avec soin, qu'on puisse 
porter quelque crédit à cette façon de se procurer une caséine de richesse connue en matière grasse (1).

\section{Mode opératoire (sur un exemple pratique).}

Un volume connu $\left(8.800 \mathrm{~cm}^{3}\right)$ de lait écrémé, dont la teneur en matière grasse $(0,8$ par litre) a été déterminée (2) par la méthode décrite précédemment [10], est abandonné au caillage spontané à douce température. Matière grasse totale mise en jeu : 7 gr. 04.

La caillebotte est traitée comme dans une fabrication de caséine (température, brassage...), puis égouttée sur de fines toiles, pressée par essorage au Büchner, lavée, pressée à nouveau. On recueille $925 \mathrm{gr}$. de caillebotte humide $(71,36 \%$ d'eau), soit $264 \mathrm{gr} .9$ de caséine sèche théorique. Après les prélèvements pour dosages d'humidité, on met la caillebotte à sécher. On procède comme dans l'industrie et on recueille finalement la caséine sèche, que l'on broye et met en flacons. (Au moment des dosages de matière grasse, l'humidité restante est bien entendu dosée).

On a mis de côté avec soin tout le sérum, les eaux de lavage,jus de pressage; leur teneur en matière grasse est déterminée comme celle du lait écrémé. Le poids total de matière grasse ainsi perdue pour la caillebotte est de $1 \mathrm{gr} .8$ environ.

Les toiles de filtration, les vases, sont séchés et rincés à l'éther (que l'on filtre avant de l'évaporer). On recueille ainsi $0 \mathrm{gr}$. $08 \mathrm{de}$ matière grasse.

La matière grasse totale perdue s'élève à $1 \mathrm{gr}$. 88 environ.

La matière grasse passée (et retenue) dans la caillebotte est donc égale à : $7,04-1,88=5$ gr. 16 environ.

Le poids total de caillebotte (supposée sèche) étant de 264 gr. 9, la teneur théorique de la caséine sèche est de 1 gr. 96 pour cent environ.

La caséine produite contenant encore $5 \%$ d'eau, le produit final sur lequel nous travaillerons titre environ 1,87 \% (soit 1,9\% en chiffres ronds).

Nos autres bilans ont été faits de la même façon. Nous avons ainsi fabriqué quelques caséines, dont voici les teneurs a priori :

\begin{tabular}{|c|c|}
\hline Caséine du bilan $n^{\circ} 1$ : env & 1,90 \\
\hline 9 du bilan $n^{\circ} 2$ : environ...$\ldots \ldots \ldots$ & 2,00 \\
\hline Caséine du bilan $n^{\circ} 3$ : environ $\ldots \ldots \ldots \ldots$ & 2,10 \\
\hline Caséine du bilan $n^{\circ} 4$ : environ ... & 2,20 \\
\hline Caséine du bilan $n^{\circ} 5$ : environ ... & 2,20 \\
\hline
\end{tabular}

(1) D'ailleurs la méthode d'analyse qui sera choisie ne sera pas basée sur ce seul critérium puisque nous essaierons d'apporter des preuves directes de son exactitude. Mais si ees deux faisceaux de preuves conduisent au même résultat, nous pourrons en conclure que la méthode d'analyse choisie est bonne.

(2) Sur 5 prises différentes ayant donnó le même chiffre. 
Remarque. - Nous avons également fait des bilans en titrant le lait écrémé, les sérums et eaux de lavage par la méthode d'Adam. Les chiffres obtenus en matière grasse mise en œuvre et en matière grasse perdue sont un peu trop forts, comme nous l'avons démontré. Mais la différence n'est pas très considérablement affectée, en sorte que le chiffre théorique obtenu pour la caséine est assez voisin de celui que l'on obtient dans un bilan suivi avec notre butyromètre. Avec la caséine donnée ci-dessus en exemple (bilan no 1 ), nous avons obtenu $1,70 \%$ de teneur théorique sur produit séché au lieu de 1,87\%. Nous n'avons retenu pour tous nos bilans que les chiffres donnés par la méthode type Gerber que nous avons décrite dans un précédent travail.

Ayant ainsi en mains un certain nombre de caséines à teneur en matière grasse connue a priori avec une approximation suffisante, nous allons les soumettre à l'analyse.

\section{*** \\ DEUXIEMME PARTIE.}

\section{CHOIX D'UNE METHODE DE BASE DONNANT UN CHIFFRE EXACT.}

Nous avions naturellement le choix entre les méthodes par extraction à l'éther et les méthodes du type Gerber. Mais l'exactitude obtenue dans une méthode d'extraction pouvant provenir d'une compensation entre les pertes dues à une extraction incomplète et les surcharges dues à d'autres matières entraînées, nous avons préféré chercher tout de' suite dans les méthodes du type Gerber (qui ne s'exposent, elles, qu'à donner des chiffres trop faibles par insuffisance de séparation).

Ene méthode du type Gerber possède deux critériums d'exactitude, que nous connaissons déjà :

$1^{\circ}$ Il est avéré que la méthode Gerber appliquée aux laits n'altère pas la matière grasse, ou en tout cas n'en fausse pas le volume. Nous avons démontré [10] que même en exagérant dans une certaine mesure la température, la concentration de l'acide ou la durée de contact avec l'acide, on n'augmente pas le volume de la matière grasse séparée dans les butyromètres. Donc les méthodes du type Gerber ne risquent pas, semble-t-il, de pécher par excès.

$2^{\circ}$ D'autre part, elles peuvent donner des chiffres trop faibles si on s'adresse à des substances très pauvres. Mais en prolongeant suffisamment la durée de contact et en centrifugeant davantage, on parvient à accroître le taux de matière grasse jusqu'à un maximum qui ne varie plus. Nous avons ainsi démontré que dans les produits pauvres en matière grasse (comme le lait écrémé), il faut chercher à obtenir un chiffre constant, chiffre alors exact. 
Nous avons pensé que ce critérium d'exactitude de la méthode Gerber (critérium que nous ne possédons pas pour les méthodes d'extraction) pourrait être appliqué au cas des caséines. C'est pourquoi nous avons cherché la méthode de base dans le groupe des méthodes du type Gerber.

§ I. CRitique des méthodes actuellement en usage. Nous savons que plusieurs expérimentateurs ont déjà mis au point pour leur propre usage, ou adapté la méthode Gerber au cas des caséines. Il est possible qu'ils soient parvenus à des résultats parfaits. Nous ne pouvons parler ici que des méthodes qui ont été publiées et nos critiques ne s'adressent qu'à elles. Les deux principales, dont découlent d'ailleurs les autres, sont dues à KRUGER et à TAPERNOUX (ce dernier s'inspirant du procédé Teichert pour les poudres de lait).

\section{Méthode Kruger.}

a) Mode opératoire de Kruger [7]. - Dans un butyromètre Gerber ordinaire à lait entier, on introduit $10 \mathrm{~cm}^{3}$ d'acide sulfurique Gerber, $9 \mathrm{~cm}^{3}$ d'eau, $1 \mathrm{~cm}^{3}$ d'alcool amylique et $2 \mathrm{gr}$. de caséine. On bouche, on agite et on porte au bain-marie à $60^{\circ}-70^{\circ}$ en agitant de temps en temps. Après dissolution, on laisse encore au bain-marie pendant 10 minutes environ. On centrifuge 15 minutes, on replace 10 minutes à $60^{\circ}-70^{\circ}$. On multiplie le volume de matière grasse par 0,55 , puis par $1,03(1)$. On obtient ainsi le pourcentage de matière grasse de la caséine.

b) Résultats obtenus avec cette méthode. - En appliquant à la lettre la méthode Kruger aux caséines que nous avons préparées, nous trouvons les résultats suivants :

\begin{tabular}{|c|c|c|c|c|c|}
\hline \multirow[t]{2}{*}{ 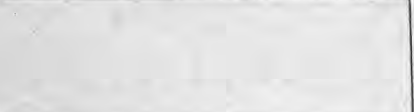 } & \multicolumn{5}{|c|}{ Nos des caséines } \\
\hline & 1 & 2 & 3 & 4 & 5 \\
\hline & environ & environ & environ & environ & environ \\
\hline Chiffres de nos bilans ........ & 1,9 & 3,0 & 2,1 & 2,2 & 2,2 \\
\hline $\begin{array}{l}\text { Méthode Kruger : } \\
\text { Io Nombre de divisions au } \\
\text { butyromètre normal .... }\end{array}$ & 3 & 3 & 3,5 & 3,5 & 3 \\
\hline $2^{\circ}$ Pourcentage calculé selon & & & & & \\
\hline KRUGER $\ldots \ldots \ldots \ldots$ & 1,6 & 1,6 & 1,9 & 1,9 & 1,6 \\
\hline
\end{tabular}

(1) Parce qu'une division, correspondant à $1 \mathrm{gr}$. de matière grasse par litre de lait, équivaut à la matière grasse absolue de $11 \mathrm{~cm}^{2}$ de lait, soit $11 \mathrm{mgr}$. Le résultat pour les caséines étant obtenu sur $2 \mathrm{gr}$. de produit, il faut pour amener ì 100 , multiplier par $50(0,011 \times 50=$ 0,55 ). Le coefficient 1,03 ne s'applique qu'aux tubes gradués au kilo. 
On trouve done des chiffres trop faibles par rapport à la teneur théorique, supposée exacte, de nos caséines. En outre ces ehiffres ne peuvent être garantis, l'erreur possible étant d'une demi-division (au moins) ou 0,3 en matière grasse pour cent. Ces écarts suffiraient presque à faire condamner la méthode Kruger. Mais d'autres arguments viennent la faire rejeter.

c) Critique de la méthode Kruger. - Le reproche le moins grave que l'on puisse faire à cette méthode est son manque de précision. En effet une caséine qui titre 1,5\% de matière grasse ne donne que 3 divisions au butyromètre Gerber normal. L'erreur absolue possible avec cet appareil est, disions-nous, d'au moins une demi-division, ee qui entraîne ici une erreur absolue d'au moins $15 \%$. La pratique nous a montré que cette erreur pouvait atteindre le double.

Avant de découvrir la cause d'erreur la plus grave de la méthode Kruger, nous avions songé à la perfectionner d'abord par l'utilisation d'un appareil plus précis. L'appareil que nous avons créé pour l'analyse des laits écrémés a été conçu pour être en même temps utilisé au dosage de la matière grasse des caséines. Toutes les critiques que nous avons faites des butyromètres courants à lait écrémé et du butyromètre normal appliqué aux laits écrémés, pourraient être reproduites ici au sujet de l'usage qu'on peut faire des uns et des autres à l'analyse des caséines. En utilisant le mode opératoire de Kruger avec cet appareil, nous avons évidemment obtenu plus de précision, mais pas davantage de résultats satisfaisants. La raison en est la suivante : quand après avoir terminé le dosage selon KRUGER, on soumet le butyromètre à un repos prolongé et à de nouvelles centrifugations, on s'aperçoit que le volume de la matière grasse augmente jusqu'à un maximum qui ne varie plus.

Nous avons apporté la preuve [10] que l'extraction de petites quantités de matière grasse dans la méthode Gerber (qu'il s'agisse de laits écrémés ou même de matière grasse pure introduite en quantité connue dans le butyromètre), exige plus d'une simple centrifugation. Il en faut de nombreuses pour extraire la totalité de la matière grasse et il est nécessaire de les combiner avec des temps de repos suffisants. Nous avons montré également que l'excès de centrifugation et l'excès de la durée de l'essai n'ont aueune influence désavantageuse sur le chiffre de matière grasse, lequel ne varie plus quand le maximum est atteint.

Toute cette discussion trouverait sa place ici dans le cas des caséines. Les conclusions sont les mêmes en ce qui concernel'influence des centrifugations répétées et des repos. Cela explique aisément que l'on ne trouve pas le chiffre constant (c'est-à-dire le chiffre exact) avec la centrifugation unique du mode opératoire de Kruger. Voici 
d'ailleurs les chiffres obtenus par la méthode Kruger initiale et ceux que l'on obtient en cherchant à atteindre le chiffre constant :

\begin{tabular}{|c|c|c|c|c|c|}
\hline \multirow{2}{*}{ 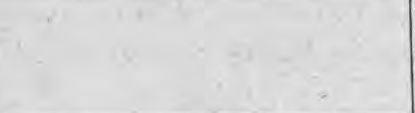 } & \multicolumn{5}{|c|}{ Nos des caséints } \\
\hline & 1 & 2 & 3 & 4 & 5 \\
\hline $\begin{array}{l}\text { Chiffres approximatifs des bi- } \\
\text { lans } \ldots \ldots \ldots \ldots \ldots \ldots \ldots\end{array}$ & 1,9 & 2,0 & 2,1 & 2,2 & 2,2 \\
\hline $\begin{array}{l}\text { Méthode Kruger (dans le buty- } \\
\text { romètre normal) (1 centrifu- } \\
\text { gation) } \ldots \ldots \ldots \ldots \ldots \ldots\end{array}$ & 1,6 & 1,6 & 1,9 & 1,9 & 1,6 \\
\hline $\begin{array}{l}\text { Méthode Kruger ( } 1 \text { centrifuga- } \\
\text { tion) dans notre butyro- } \\
\text { mètre } \ldots \ldots \ldots \ldots \ldots \ldots \ldots\end{array}$ & 1,50 & 1,65 & 1,80 & 1,75 & 1,80 \\
\hline $\begin{array}{l}\text { Centrifugations jusqu'à chif- } \\
\text { fres constants à la suite du } \\
\text { Kruger } \ldots \ldots \ldots \ldots \ldots \ldots\end{array}$ & 1,95 & 2,05 & 2,10 & 2,15 & 2,15 \\
\hline
\end{tabular}

Les écarts entre le Kruger (dans notre tube) et la centrifugation à chiffre constant, atteignent 0,4 de matière grasse pour cent de caséine.

Remarque. - Un point toutefois restait à préciser qui n'avait pas fait l'objet de recherches dans notre étude sur les laits écrémés : le maintien prolongé à la température de $67^{\circ}$. Pour les laits écrémés, en effet, où ce séjour au bain-marie n'excède pas 10 minutes en deux fois (les autres repos se faisant à la température ordinaire), nous avions constaté que la matière grasse ne subissait aucune altération (chiffre de matière grasse introduite retrouvé). Ici, la dissolution de la caséine exige un séjour préalable prolongé en tube au bain-marie. Nous pouvions nous demander si à $67^{\circ}$ pendant une heure (durée nécessaire à la dissolution dans le Kruger) la matière grasse ne subirait pas une altération qui serait de-nature à en diminuer ou en augmenter le volume. Il n'en est rien. On retrouve également dans ces conditions, même après 4 heures de contact à $67^{\circ}$, le chiffre de matière grasse pure introduite.

Notons en passant que si le contact est prolongé 4 heures au lieu d'une, l'extraction de la matière grasse se fait beaucoup plus aisément. On arrive plus vite au chiffre exact (une ou deux centrifugations au maximum) après un contact de 4 heures qu'après un contact d'une heure. Ce point est une confirmation de la nécessité, déjà démontrée, des repos, même pour l'extraction de la matière grasse pure. 
Les écarts de 0,4 observés ci-dessus ne sont done bien dus qu'à une insuffisance d'extraction dans le Kruger, et non pas à une altération de la matière grasse.

En résumé: La méthode Kruger ne peut être conservée :

10 Parce qu'elle utilise un butyromètre à lait entier trop imprécis ;

$2^{\circ}$ Parce que même avec un butyromètre précis elle donne des chiffres trop faibles, dus à une insuffisance d'extraction de la matière grasse.

\section{$2^{\circ}$ Méthodes Type Teichert (Schoojans, Tapernoux).}

La méthode Teichert pour le dosage de la matière grasse des poudres de lait a été appliquée au cas des caséines par ScHOONJ ANS [9] puis par TAPERNoux [8]. Elle diffère de la méthode Kruger par l'emploi d'une température plus élevée pour la dissolution de la caséine $\left(85^{\circ}\right)$.

a) Mode opératoire de cette méthode. - Dans un butyromètre à poudre de lait on introduit $10 \mathrm{~cm}^{3}$ d'acide sulfurique Gerber, $8 \mathrm{~cm}^{3}$ d'eau, 2 gr. 5 de caséine et $1 \mathrm{~cm}^{3}$ d'alcool amylique. On maintient au bain-marie à $85^{\circ}-90^{\circ}$ pendant 2 heures en ayant soin de retourner de temps en temps le butyromètre. On centrifuge 3 minutes à 1.200 tours et on effectue la lecture à $65^{\circ}$.

b) Résultats obtenus :

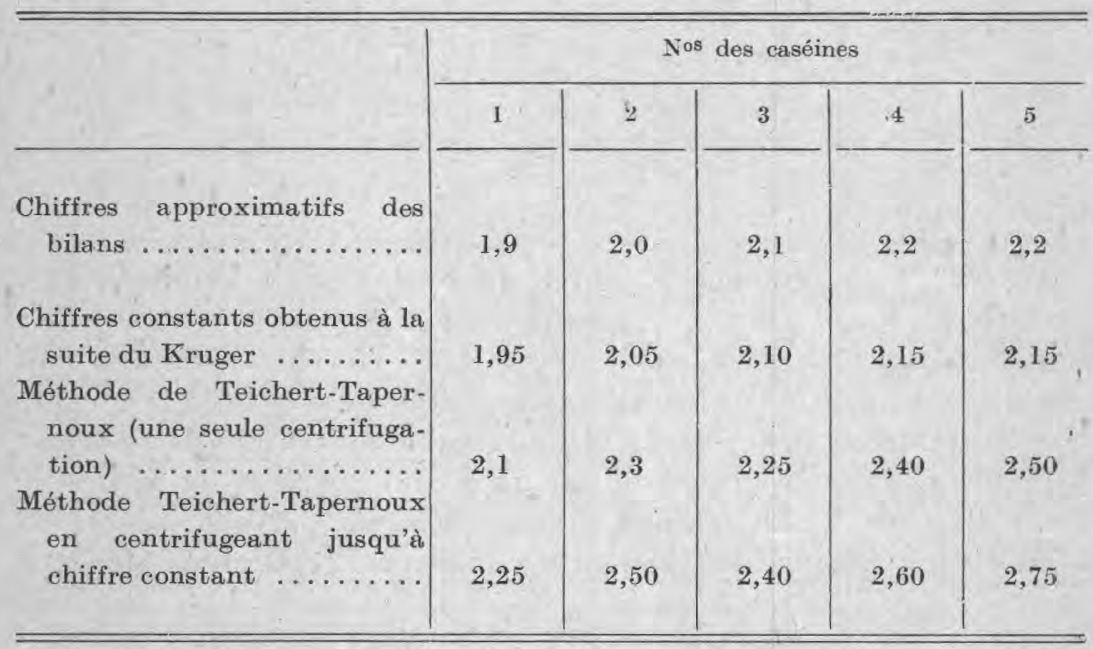

On trouve cette fois des chiffres trop élevés par rapport à la teneur exacte déjà donnée par le Kruger amélioré. 
La certitude que nous croyons avoir acquise de l'exactitude des chiffres constants suffirait, semble-t-il, à nous faire condamner la méthode Teichert.

Nous avons cependant cherché à découvrir la cause de ces écarts pour le moins inattendus.

c) Gritique de la méthode Teichert-Tapernoux. - Sans décrire tous les essais infructueux que nous avons d'abord effectués dans ce domaine, indiquons seulement l'hypothèse qui nous a finalement mis sur la voie :

Dans les laits écrémés, rappelions-nous ci-dessus, les températures de $67^{\circ}$ et même de $85^{\circ}$ maintenues seulement 10 minutes en deux fois, n'altèrent pas le chiffre de matière grasse séparée. En est-il de même quand le contact se prolonge deux heures?

Nous venons de dire qu'on n'observe pas d'altération à $67^{\circ}$. Il restait à voir, à l'occasion de cette méthode, ce qui se passerait à $85^{\circ}$. Or voici ce que nous avons observé :

a) A $85^{\circ}$ pendant 2 heures la matière grasse s'altère par accroissement de volume. Matière grasse pure introduite : $50 \mathrm{mgr}$., soit 25 petites divisions.

Quatre séries de tubes sont maintenus respectivement 5 minutes, 45 minutes, 90 minutes et 3 heures à $85^{\circ}$, puis centrifugées jusqu'à chiffre constant. de petites divisions)

\begin{tabular}{r|rrrr}
5 minutes & 25,5 & 25,0 & 25,5 & 25,5 \\
45 minutes & 24,5 & 25,0 & 25,5 & 25,0 \\
90 minutes & 31,0 & 30,5 & 33,0 & 31,5 \\
3 heures & 35,0 & 33,5 & 35,5 & 34,0
\end{tabular}

Donc, si on prolonge la durée du contact avec l'acide (à $85^{\circ}$ ) au delà de 45 minutes, la quantité de matière grasse extraite par centrifugation à chiffre constant est trop élevée, supérieure à la quantité introduite. (Les lectures sont toujours faites à $65^{\circ}$.) Ce phénomène pourrait peut-être s'expliquer par une fixation de radicaux $\mathrm{SO}^{4}$ sur la molécule grasse ou par un commencement de décomposition de celle-ci. La matière grasse est assez souvent colorée en brun plus ou moins foncé dans ces conditions. (1) Notons bien que

(1) La matière grasse pure utilisée était du beurre pur, lavé à fond à chaud, filtré, séché, dissous dans l'éther. D'autres essais semblent nous avoir apporté la preuve que cette altéra. tion est d'autant plus intense que la matière grasse utilisée provient de substances ayant subi une fermentation lactique plus avancée. L'état de nos travaux dans ce domaine ne nous permet pas d'apporter plus de précisions, mais nous nous réservons de revenir sur cette question dans un autre but. 
ces résultats ne sont pas obtenus avec le mode opératoire TeichertTapernoux, qui se borne à une seule centrifugation (d'où cause de déficit par ailleurs), mais bien jusqu'à chiffre invariable correspondant à une extraction complète de la matière grasse. Nous avons observé ce phénomène d'une manière régulière sur des matières grasses pures (de beurre) d'origines différentes. Nous pensons pouvoir dire qu'il est général, au moins sur les matières grasses provenant de beurres, crèmes, caséines : substances qui ont subi une fermentation lactique préalable.

ß) La caséine pure, soumise aux mêmes influences, ne subit pas d'altération de nature à fausser le volume de la matière grasse.

Bien que l'observation précédente, parfaitement et régulièrement confirmée, soit suffisante pour condamner la méthode Teichert pour la caséine (et les méthodes similaires utilisant $85^{\circ}$ pour la dissolution de la caséine), il nous a paru intéressant de chercher si la matière protéique elle-même (caséine pure) ne subirait pas à $85^{\circ}$ pendant plusieurs heures une altération susceptible de libérer des substances pouvant être séparées comme de la matière grasse et capables par conséquent d'accroître le volume apparent de la matière grasse véritable.

Nous avons préparé de la caséine pure de la façon suivante. Plusieurs dizaines d'extraction éthérées (selon ADAM) furent effectuées sur un lait écrémé. La eouche inférieure hydroalcoolique (renfermant caséine, lactose, sels) fut recueillie, débarrassée de l'alcool et des traces d'éther au bain-marie, puis précipitée par l'acide acétique. La caséine obtenue fut séparée, lavée, séchée. Etant donné la valeur du procédé d'Adam comme moyen d'extraction de la matière grasse du petit-lait (nous en avons refait la démonstration dans une étude précédente), nous sommes fondés à penser que l'extrait hydroalcoolique et, donc, la caséine qui en provient ne contiennent pas de matière grasse.

En soumettant cette caséine aux modes opératoires Kruger et Teichert, nous n'avons observé aucune séparation de substances oléiformes dans le butyromètre après dissolution de 2 heures à $65^{\circ}$ ou à $85^{\circ}$ et centrifugations prolongées, entrecoupées de repos de 12 ou de 24 heures.

$\gamma$ ) Le lactose, soumis aux mêmes influences, abandonne une substance oléiforme.

Le lactose pur soumis au contact de l'acide Gerber et de l'alcool amylique dans les conditions d'un dosage de matière grasse, donne à $85^{\circ}$, après 2 heures de séjour et centrifugation, une matière huileuse colorée qui peut se confondre avec de la.matière grasse. A $67^{\circ}$ pendant le même temps, il ne se forme que des traces insignifiantes de cette substance. En opérant sur de petites quantités de lactose 
(de 5 à $10 \mathrm{mgr}$. dans le butyromètre), comme celles qui peuvent se rencontrer dans $2 \mathrm{gr}$. de caséine mal lavée, on observe une séparation de matière huileuse qui occupe les volumes suivants :

Après 3 heures de contact à $65^{\circ}$ et centrifugation à chiffre constant : néant ou traces (moins d'une demi-petite division).

Après 3 heures de contact à $85^{\circ}$ et centrifugation à chiffre constant : 0,30 à 0,40 ( 3 à 4 petites divisions).

Une dizaine d'essais analogues ont confirmé ce résultat. Il semble même que la quantité de matière oléiforme formée ne varie guère quand on augmente notablement la quantité de lactose introduit. Nous n'avons pas cherché l'explication de ce phénomène, dont l'observation est absolument certaine et constante. Bien entendu, cette cause d'erreur ne jouerait que sur des caséines contenant une certaine quantité de lactose $(2 \%)$ et n'aurait que peu d'influence dans le cas de caséines bien lavées.

En résumé: Les méthodes du type Teichert, Schoonjans, Tapernoux semblent devoir être abandonnées, tout au moins pour les caséines, parce qu'à $85^{\circ}$ pendant le nombre d'heures indiqué par ces auteurs, on constate une altération de la matière grasse et du lactose qui augmente le volume apparent de la matière grasse séparée (1). Rappelons que ces altérations ne se produisent pas à $67^{\circ} \mathrm{en}$ 1 à 2 heures, ni même en quelques minutes à $85^{\circ}$.

\section{\$ II. MISE AU POINT D'UNE MÉthOdE dE dOSAGE EXACT DE LA} MATIERE GRASSE DES CASÉINES. - Tout ce que nous venons de dire nous conduit, semble-t-il, à reconnaître que la solution du problème consiste à a méliorer la méthode Kruger (dissolution à $65^{\circ}$ ) :

par l'emploi d'un appareil précis et adapté à son but,

par l'emploi d'une technique simple permettant d'atteindre aisément le chiffre exact.

$1^{\circ}$ Appareil. - Le butyromètre spécial que nous avons créé (2) pour les laits écrémés convient très bien au dosage des caséines. En opérant sur $2 \mathrm{gr}$, la totalité de l'échelle correspond à $5 \%$ de matière grasse dans la caséine. L'une des 5 grandes divisions représente $1 \%$ et chaque petite division $0,1 \%$ de matière grasse.

$2^{\circ}$ Technique. - Diverses questions de techniques ont dû être préalablement résolues. Voici les conclusions partielles auxquelles nous avons abouti :

(I) Nous passons sous silence le fait que la méthode Teichert (une seule centrifugation) donnerait par ailleurs un chiffre trop faible en raison de l'insuffisance de l'extraction de la matière grasse. Il en résulte que la cause de surcharge due aux altérations pourrait, dans certains cas, être compensée par cette erreur en moins. Mais on ne saurait fonder une méthode sur l'espérance d'une compensation d'erreurs aléatoire.

(2) Butyromètre à laits écrémés et caséines, modèle des "Fermiers Réunis v, construit par la maison Gerber, Sihlquai, 55, Zurich 5 (Suisse). 
«) L'alcool amylique doit être présent pendant la dissolution. Si on effectue la dissolution de la caséine dans le mélange eau-acide, soit en dehors du butyromètre (eapsule de porcelaine au bain-marie), soit dans le tube lui-même, l'extraction de la matière grasse se fait ensuite beaucoup moins aisément. Il faut prolonger les repos et multiplier les centrifugations pour obtenir le chiffre que l'on atteint-, par exemple, après, seulement deux centrifugations et une nuit de repos quand l'alcool amylique est introduit au moment de la dissolution. Quand la constance est atteinte dans l'un des cas, le déficit est dans l'autre de l'ordre de 0,2 à 0,3 de matière grasse pour cent de caséine.

ß) On a eherché à accélérer la dissolution à $65^{\circ}$ en faisant préalablement gonfler la caséine dans l'eau à cette température avant d'introduire l'acide et l'alcool. En fait, la dissolution est un peu plus rapide (1/4 d'heure de gain seulement) ; en revanche l'extraction de la matière grasse, au moins au début, semble moins aisée. Les premières centrifugations donnent des chiffres plus faibles. Comme, d'autre part, eette opération compliquerait le dosage, nous l'avons abandonnée.

Inversement, il n'y a aucun avantage à dissoudre la caséine dans de l'acide plus concentré que le mélange à volumes égaux acide Gerber et eau. La dissolution est plus lente et la matière grasse fortement colorée.

ү) Il est préférable d'effectuer la dissolution de la caséine dans le butyromètre lui-même, plutôt que dans une capsule. Cette façon d'opérer est rendue utile, voire nécessaire, par l'intérêt de la présence de l'alcool amylique au moment de la dissolution, par l'avantage d'agitations violentes qui ne peuvent se faire qu'en tube bouché, par la suppression d'une cause de pertes possibles aux transvasements et par la simplification du mode opératoire. En procédant ainsi il arrive que des grains de caséine passent dans le canal gradué et s'y fixent momentanément. Il n'y a pas lieu de s'en émouvoir, car ils disparaissent sans difficulté au cours de la dissolution au bainmarie.

Nous avons également adopté le mélange à parties égales d'acide sulfurique Gerber et d'eau pour les raisons qui ont été données antérieurement [10].

i) Comme pour les laits écrémés, nous avons observé qu'un repos prolongé à la température ordinaire est nécessaire et remplace avantageusement de nombreuses centrifugations. Pour les caséines, une nuit de repos au moins est indispensable dans tous les cas. Après ce repos une nouvelle centrifugation est également à prescrire, à l'inverse de ce qui se passe pour les laits écrémés. Dans le cas des easéines il y a lieu de tenir compte, en effet, d'une plus grande 
viscosité de la masse due à la mise en solution de 2 gr. de matière, alors qu'avec $20 \mathrm{~cm}^{3}$ de lait écrémé on n'apporte que $0 \mathrm{gr}$. 6 de caséine environ.

Ces différentes observations, jointes à l'étude précédente, nous ont amenés à préconiser le mode opératoire suivant :

§ III. MODE OPÉRATOIRE PROPOSE. - Dans un butyromètre (d lait écrémé et caséine, modèle des "Fermiers Réunis ") on introduit dans l'ordre : .

$$
\begin{aligned}
& 20 \mathrm{~cm}^{3} \text { d'acide Gerber ; } \\
& 20 \mathrm{~cm}^{3} \text { d'eau ; } \\
& 2 \mathrm{gr} \text {. de caséine (1) ; } \\
& 1 \mathrm{~cm}^{3} \text { d'alcool amylique. }
\end{aligned}
$$

On bouche avec un bouchon Gerber classique dont on aura sectionné 5 m/ de l'extrémité utilisée pour en diminuer le volume (2). On retourne plusieurs fois le tube et on l'agite vigoureusement ; on le porte au bain-marie réglé à $65^{\circ}-70^{\circ}$ jusqu'à dissolution complète (et on $l^{\prime} y$ laisse encore 10 minutes) en ayant soin de retourner et d'agiter le tube de temps en temps (3).

La dissolution étant achevée, on centrifuge une première fois à 1.200 tours pendant 5 minutes.

On porte 5 minutes au bain-marie à $67^{\circ}$ en ayant soin de procéder au réglage du bouchon comme il a été indiqué pour les laits écrémés (Le Lait, 1932, p. 395). Le niveau supérieur de la matière grasse doit se trouver (à la fin du séjour au bain-marie) vers le tiers inférieur de l'échelle graduée. De cette façon, la mise en place de la matière grasse pour la lecture finale pourra se faire automatiquement par le jeu de la dilatation dés liquides sans avoir à manceuvrer le bouchon, ce qui est à éviter.

Ce réglage étant fait, on procède à une deuxième centrifugation ; et le tube est abandonné au repos, au sortir de la centrifugeuse, à la température ordinaire pendant 24 heures.

Ce délai nécessaire étant révolu, on porte le tube au bain-marie à $67^{\circ}$ pendant 5 minutes; on centrifuge 5 minutes à 1.200 et on porte à nouveau au bain-marie à $67^{\circ}$ pour effectuer la lecture.

Celle-ci se fait aisément, puisque au bout de 5 minutes environ à cette température, la matière grasse vient se placer d'elle-même.

(I) Il n'est pas nécessaire que la caséine soit finement broyée. On peut même utiliser des gruaux passant au tamis $n^{\circ} 30$.

(2) Cette nécessité nous ést imposée par l'affectation d'un même appareil 'à deux usages. différents où le volume tótal des matières n'est pas le même. Elle peut être remplacée par l'introduction de $19 \mathrm{~cm}^{3}$ d'eau au lieu de 20 sans ineonvénient.

(3) La dissolution d'un gruau exige environ 50 minutes ; celle d'une farine 25 à 30 minutes. On paut adopter une heure dans tous les cas, sous réserve d'avoir fréquemment retourné les tubes. 
dans la colonne graduée et avec une lenteur suffisante pour permettre la surveillance et la lecture d'une dizaine de tubes. Il faut éviter de manceuvrer le bouchon pour la lecturé, car les montées ou les descentes trop rapides de la matière grasse dans le tube en tapissent les parois et faussent le dosage. Le pourcentage est donné directement. Les grandes divisions représentent les unités. Les petites divisions représentent les dixièmes. Ainsi 22 petites divisions correspondent à une caséine titrant 2,2\% de matière grasse. Les écarts possibles en utilisant cette technique, entre des butyromètres et des opérateurs différents, ne dépassent pas 0,1 de matière grasse pour eent d'une caséine donnée.

Remarques : $1^{0} \mathrm{Si}$, malgré tout, on conserve un doute sur le résultat (ou dans le but d'obtenir une confirmation de l'excellence des manipulations), on peut soumettre l'essai à un nouveau repos de 12 heures et à une nouvelle centrifugation. En opérant ainsi nous n'avons jamais été amenés à majorer nos chiffres primitifs de plus de $0,1 \%$ (une petite division). Et dans la grande majorité des cas, le chiffre obtenu en suivant à la lettre la technique précédente (avec 24 heures de repos) est bien un chiffre constant, définitif, qui ne varie plus.

$2^{\circ}$ On remarquera le parallélisme étroit qui existe entre ce mode opératoire et celui que nous avons mis au point pour les laits écrémés. Il peut être utile, pour la pratique, de mettre ce parallélisme en évidence :

Laits éerémés

Introduire dans le butyromètre :

$20 \mathrm{em}^{3}$ d'acide Gerber ;

$20 \mathrm{~cm}^{3}$ dear ; de lait énewé

$2 \mathrm{~cm}^{\mathrm{n}}$ d'alcool amylique.

Boucher, retourner et agiter.

Porter au bain-marie 5 minutes à $67^{\circ}$.

Centrifuger 5 minutes à 1.200 tours.

Porter au bain-marie 5 minutes à $67^{\circ}$.

Procéder au réglage du bouchon.

Centrifuger 5 minutes à 1.200 tours.

Laisser reposer 3 heures à température ordinaire.

Porter au bain-marie 5 minutes à $67^{\circ}$.

Effectuer la lecture.

Caséines

Introduire dans le butyromètre : $20 \mathrm{~cm}^{3}$ d'acide Gerber ; $20 \mathrm{~cm}^{3}$ d'eau ;

2 gr. de caséine ;

I $\mathrm{em}^{3}$ d'aleool amylique.

Boucher, retourner et agiter (bouchon sectionné à $5 \mathrm{~m} / \mathrm{m}$ de l'extrémité).

Porter au bain-marie 1 heure à $6 \%$ en retoumant fréquemment les tubes.

Centrifuger 5 minutes à 1.200 tours.

Porter au bain-marie 5 minutes à $67 \%$.

Procéder au réglage du bouehon.

Centrifuger 5 minutes à 1.200 tours.

Laisser reposer 24 heures à température ordinaire.

Porter au bain-marie 5 minutes kो $67^{\circ}$.

Centrifuger 5 minutes à 1.200 tours.

Remettre au bain-marie 5 minutes à $67^{\circ}$. Effectuer la lecture. 
$3^{\circ}$ Cette technique peut paraître compliquée.

Dans la pratique elle s'effectue aisément, sans difficultés, et permet des dosages en série. Nous l'utilisons journellement dans nos laboratoires depuis un an.

§ IV. BASES D'APPRÉCIATION DE LA VALEUR DE CETTE TECHNIQuE. - Les chiffres obtenus à l'aide de cette technique sont les mêmes que ceux qui ont été donnés plus haut en appliquant la méthode Kruger (avec notrę butyromètre) et en centrifugeant à chiffre constant. La comparaison qui a été faite ayec les chiffres de nos bilans constitue déjà un sérieux argument en faveur de l'exactitude de notre mode opératoire. Mais nous pouvons en outre rappeler ici les deux principes qui sont à la base de nos travaux sur l'application de la méthode Gerber aux dosages de petites quantités de matière grasse, principes qui, selon nous, justifient nos résultats :

a) L'obtention d'un chiffre maximum au Gerber ne correspond pas (à $67^{\circ}$ ) à des chiffres trop élevés; car une fois le volume vrai obtenu (sur du beurre pur par exemple), les influences mises en jeu pour l'obtenir ne le font plus varier. (Température de $67^{\circ}-70^{\circ}$, centrifugations, repos.)

Done, nos chiffres ne doivent pas pécher par excès.

D'ailleurs, ces mêmes influences ne libèrent pas plus de substances oléiformes à partir de la caséine pure et du lactose qu'elles n'altèrent le volume de la matière grasse pure introduite.

b) Ils ne doivent pas non plus pécher par défaut ; car notre mode opératoire correspond à l'obtention d'un chiffre maximum eonstant, non susceptible de varier par de nouvelles centrifugations combinées avec des repos prolongés.

Nous sommes done fondés à penser que les chiffres que nous obtenons sont exacts.

$$
* * *
$$

RÉSUMÉ ET CONCLUSIONS DE CE PREMIER ARTICLE.

1. Les nombreuses méthodes proposées pour le dosage de la matière grasse des caséines se divisent en deux groupes : méthodes d'extraction par l'éther (sur caséine dissoute) et méthodes issues du procédé Gerber.

2. Nous avons préparé des caséines à teneur en matière grasse approximativement connue et leur avons appliqué d'abord les méthodes du type Gerber dans le but d'essayer de découvrir une méthode dont l'exactitude soit plus aisément démontrable.

3. La méthode Kruger (type Gerber avec dissolution à 670) doit être rejetée : 
a) D'abord parce qu'elle utilise le butyromètre à lait entier, qui manque, ici, de précision.

b) Ensuite parce que même appliquée à l'aide d'un appareil précis, elle est déficitaire $(0,4$ à 0,5 de matière grasse pour cent de easéine) : elle ne fait appel qu'à une seule centrifugation, sans repos prolongé.

Nous avons démontré que même avøc de la matière grasse pure, il faut prolonger les centrifugations et les repos pour retrouver la quantité introduite. Pour les caséines on arrive par les mêmes procédés, à obtenir un chiffre constant sans altération de la matière grasse.

4. La méthode Teichert-Tapernoux (type Gerber avec dissolution à $85^{\circ}$ ) doit être également rejetée :

a) Parce qu'elle donne des chiffres trop forts, en raison d'une altération de la matière grasse (en 2 heures à $85^{\circ}$ ) qui a pour résultat d'en accroitre le volume, et aussi paree que le lactose abandonne dans les mêmes conditions une substance oléiforme qui majorela matière grasse (cas des caséines mal lavées).

L'altération de la matière grasse et du lactose ne se produisent pas à $67^{\circ}$ pendant 1 heure.

La matière protéique pure n'abandonne aucune substance oléiforme, ni à $67^{\circ}$ ni à $85^{\circ}$ pendant 3 heures.

b) Parce qu'en dehors de eette surcharge certaine, la méthode tend, d'autre part, à être déficitaire, puisqu'elle ne fait appel qu'à une seule centrifugation sans repos suffisant.

5. Nous avons done rejeté totalement la seconde de ces méthodes et cherehé à améliorer la première :

a) En l'exécutant avec un butyromètre précis que nous avons conçu.

b) En fixant un mode opératoire qui permet l'extraction complète de la matière grasse sans altérations susceptibles d'en fausser le volume.

Cette méthode nouvelle permet d'obtenir le résultat avec la certitude que l'erreur commise est inférieure à 0,1 de matièregrasse pour cent de caséine. Elle se prête aussi bien aux dosages industriels en série qu'aux recherches de laboratoire.

Elle se rapproche dans ses grandes lignes de la méthode antérieurement mise au point pour les laits écrémés.

$$
*^{*} *
$$

Possédant une méthode du "type Gerber " que nous croyons bonne, et ayant d'ailleurs constaté que les autres méthodes du même type sont entachées d'erreur, nous procédons sur les mêmes easéines, à des dosages par les méthodes d'extraction éthérée, pour essayer de 
découvrir celles qui sont exactes et, si possible, trouver la raison de l'inexactitude des autres.

L'exposé de ces travaux fera l'objet d'un article ultérieur.

\section{BIBLIOGRAPHIE.}

[1] H. SHAw Roscoe. Journal of Industrial and Engeneering Chemistry, 1920, $12,1169$.

[2] TAPERnoux. Le Lait, 1928, 592.

[3] Höpener. Annales des Falsifications et des Fraudes, mars 1927, 166.

[4] DoRnic et DaIrE. Revue générale du Lait, 1909, 328.

[5] Chollet. Le Lait, 1929, 267.

[6] Fou

[7] E. KRUGER. Schweizer Milchztq., 1928, 53, 369.

[8] TAPERNoUX. Le Lait, 1928, 592.

[9] Schoonjans. Le Lait, 1925, 782.

[10] Pien et MHe Madrick. Le Lait, 1932, 269.

\section{LA CONSTANTE MOLÉCULAIRE SIMPLIFIÉE APPLIQUÉE AUX LAITS DE LA LOIRE-INFÉRIEURE (1).}

par

le Dr ROBIN

Directeur du Service municipal d'Hygiène de Nantes. et

Directeur des Laboratoires d'analyse de la Biscuiterie Lefèvre-Utile.

Lorsqu'en 1906, le Professeur Porcher mit en valeur ce fait eapital pour l'analyse du lait, à savoir que les chlorures étaient le régulateur de sa pression osmotique et que leurs variations étaient inverses de celles du lactose, un grand pas venait d'être accompli dans la voie de la répression des fraudes.

En effet, en janvier 1914, MM. Mathied et Ferré publiaient dans les Annales des Falsifications, leur travail sur la Constante moléculaire simplifiée, qui découlait du principe posé par Ch. Porcher, et fixaient les limites entre lesquelles varie ce nouveau facteur d'appréciation du mouillage.

Depuis cette époque, nombre de chimistes ont publié les résultats obtenus dans leurs laboratoires par l'application de cette précieuse donnée aux analyses faites sur des laits individuels ou de mélange do leur région.

Grâce à cette documentation, nous pouvons assurer aujourd'hui que la constante moléculaire simplifiée est devenue une fidèle appréciation de la fraude du lait par mouillage, tout au moins dans la très grosse majorité des cas, et en dépit des influences, ethnologiques, climatériques, voire physiologiques, auxquelles les animaux producteurs peuvent être soumis.

(1) Paru dans le Volume jubilaire du Prafesseur Ch. Porcher. 